\title{
Effects of a Symbiotic on the Quality of Life of Elderly Patients with Breast Cancer: a Randomized Controlled Pilot Trial
}

\author{
Rossi $C^{1}$, Filippone $A^{1}$, Rossi MM ${ }^{1}$, Guarino $D^{1}$, Magno $S^{1}$, Lissoni $P^{2}$, Scarpa $S^{2}$, Marchiori $P^{2}$, Vigliar $P^{2}$, Hiroaki Maeda ${ }^{3}$ and \\ Messina $\mathrm{G}^{2}$ \\ ${ }^{1}$ Center for Integrative Oncology, Fondazione Policlinico Universitario Agostino Gemelli IRCCS, Rome, Italy \\ ${ }^{2}$ International Institute of Pnei, Milano, Italy \\ ${ }^{3}$ Japan Association for Food Functional Clinical Research, Tokyo, Japan
}

${ }^{\star}$ Corresponding author: Dr. Giusy Messina, International Institute of Pnei, Milano, Italy; Email: giusy.messina@libero.it

Received: February 01, 2021; Accepted: February 09, 2021; Published: February 15, 2021

\begin{abstract}
Background: A number of studies have confirmed the beneficial effects of prebiotics and probiotics on several physical and psychological health outcomes. The present study aims to evaluate the feasibility, tolerance and preliminary results of a symbiotic, composed by oryzalose and lactobacillis plantarum on sleep quality and psycho-physical stress in a group of elderly patients affected by hormonal dependent breast cancer.

Methods: A total of 40 patients with hystologically proven breast cancer were randomly assigned to group A (intervention) or group B (placebo). Pittsburgh Sleep Quality Index (PSQI), Short Form Health Survey (SF-36) and HADS (Hospital Anxiety and Depression Scale) were submitted to all participants, provided an informed consent, at the enrolment and 6 months later.

Results: After treatment, the group A showed the highest SF-36 physical functioning and vitality score ( $\mathrm{P}=0,01)$, the lowest bodily pain score ( $\mathrm{P}=0,01)$ compared to the placebo control group. Group A achieved a significant improvement in the quality of life for all SF-36 domains. Moreover, the intake of symbiotic led to a significant improvement of several PSQI subscales (sleep quality, sleep disturbances, daytime dysfunction). There was a non-significant increased rate of participants classified as good sleepers. Decreased levels of anxiety and depression were observed in group A, but the difference was not significant.
\end{abstract}

Conclusion: According to our results, a combination of oryzalose and a probiotic could significantly improve both physical and psychological outcomes in a population of elderly breast cancer patients, with excellent safety profiles and optimal compliance.

Keywords: Quality of life, Psychological distress, Cancer, Microbiome, Oncobiotic

\section{Introduction}

The human microbiota is gaining more and more attention in the pathogenesis and management of several cancers, including breast cancer, within a new frame of science defined oncobiotic. As regards breast cancer, microbiome seems to be relevant for at least five reasons: the impact of dysbiosis on immune competence [1], systemic inflammation [2], hormonal milieu through the so called "estrobolome" [3-5], the emotional balance (psychobiotic) [6] and breast tissue microbial composition [7]. In fact, several studies have shown that breast tissue has a distinct microbiome with particular species enriched, and somewhat related to the gut bacteria through a gut-breast axis [8-9]. The question remains whether the microbiome plays a causal role in breast carcinogenesis or is an epiphenomenon; accordingly, probiotic treatment may be protective against the incidence of cancer and at least some of cancer related side effects [10]. The microbiome can also interfere with pharmacodynamics and efficacy of some anticancer treatment protocols, including chemotherapy and immunotherapy [11-14]. In addition, achieving benefits in terms of QoL has become increasingly important in cancer treatment, with the traditional endpoint of survival deemed insufficient as the only treatment outcome [15]. Immune dysfunction leading to inflammation is the underlying mechanism that affects the patient physically and emotionally, which also has an indirect impact on social functioning [16]. Inflammation is a hall-mark of cancer as it is associated with the microenvironment of almost all tumor sites [17]. Persistent and localized inflammation can lead to the leaking of pro-inflammatory cytokines into circulation and trigger a systemic inflammatory cascade [18]. There is a consistent relationship between increasing systemic inflammation and worsening of all QoL parameters, such as global health, physical and social functioning, fatigue, pain [19]. Increased inflammation in the central nervous system also triggers behavioural co-morbidities, including depression, anxiety, fatigue, cognitive disturbances, and neuropathic pain. In the present study, we aim to study the feasibility and tolerance of a symbiotic supplement (Superbran) composed by a prebiotic molecule (Oryzalose, a polysaccharide derived from enzymatically treated rice bran with an extract of the shiitake mushrooms), in association with Lactobacillum Plantarum, a probiotic with proven efficacy in activating the cytokine TRAIL (Tumor Necrosis Factor-Related 
Apoptosis Inducing Ligand), gamma-amino butyric acid (GABA) and anthocyanin in a population of elderly breast cancer patients. The secondary endpoint of this pilot randomized controlled trial is to evaluate the effects of this supplement on quality of sleep and quality of life of a sample of elderly breast cancer patients, compared to placebo. The health-related quality of life (QoL) of cancer patients includes the subjective perception of symptoms, as well as physical, emotional, social and cognitive functions, and the side effects of hormonal treatments [20].

\section{Materials and Methods}

This is a parallel, randomized, double-blind and placebo-controlled trial carried out at Fondazione Policlinico Universitario A. Gemelli IRCCS, Center for Integrative Oncology, in Rome and at International Institute of Psychoneuroendocrineimmunology (PNEI) in Milan. A total of forty (40) non-metastatic female patients, over the age of 65 (median age: 71 years, range 65-83), with histologically proven hormone-sensitive breast cancer ( $\mathrm{ER}+$ and/or $\mathrm{PR}+$ ), undergoing adjuvant hormonal therapy (aromatase inhibitors) were recruited from May 2018 to December 2019. Only three (3) of total 40 patients did not finish the study due to previous comorbidities. Twenty (20) patients were randomly assigned to the intervention group (A) and twenty (20) to the placebo group (B), matched by age and performance status. Informed consent was obtained from all the patients. The eligibility criteria were as follows: histologically proven hormonal responsive breast cancer, no ongoing corticosteroids therapy due to their immunosuppressive effects, and no concomitant treatment with other immunomodulating agents, such as interferons, interleukins and monoclonal antibodies. In both arms, supplement and placebo, supplied by PneiPharma (Milan, Italy), were administered orally in a three times/day dose for six months. At the enrolment, patients were asked to collect venous blood at 0 (baseline) and 6 months later, in the morning after an overnight fast. In each blood sample, we counted lymphocytes, monocytes and some lymphocyte subpopulations, including TH lymphocytes (CD4), cytotoxic T lymphocytes (CD8), $\mathrm{T}$ reg (CD4+CD25+), NK cells (CD16+CD56). Data were reported as mean \pm SE, and statistically analyzed by the Chiquare test, the Student's test, and the coefficient of correlation, as appropriate. Moreover, we measured patients' symptoms of depression and anxiety using the Hospital Anxiety and Depression Scale (HADS), including 14 items rated on a 4-point Likert-type scale (higher scores indicate more severe symptoms). The PSQI (Pittsburgh Sleep Quality Index Malay Version) is a standardized, self-administered questionnaire that evaluates retrospective sleep quality and disturbances within the past month. It includes 19 items forming seven subscales: (1) sleep quality (1 item), (2) sleep latency (2 items), (3) sleep duration (1 item), (4) sleep efficiency (3 items), (5) sleep disturbance (9 items), (6) sleep medication (1 item), and (7) daily dysfunction (2 items). The PSQI was evaluated following the original scoring system. Each component has a score ranging from 0 to 3 . The scores of seven components will be added up to get a total PSQI score ranging from 0 to 21 . Respondents with an overall score above 5 are classified as 'poor sleepers', while those with a score of 5 or below are classified as 'good sleepers. The SF-36 (Short Form Survery) measures 8 QOL domains which are dichotomized in physical (functioning, physical role limitations, pain, general health) and mental health (vitality, social functioning, emotional role limitations and emotional/mental health) [21]. Item scores were converted to a scale of 0-100 points; the domain scores were derived by averaging individual items within the subscale; and physical and mental health composite scores were derived by averaging the four component domains of each one. Higher values are indicative of better QOL.

All the questionnaires were administered at 0 (baseline), 3 and 6 months in all the participants.

\section{Results}

The patients both in the intervention and in the placebo group tolerated well the treatment, did not report any remarkable side effect and only three drop out was recorded. The clinical characteristics of the evaluable patients are reported in Table 1. A clear relief from asthenia was achieved by patients enrolled in the intervention group (83\%).

Improvements in QOL (SF-36) scores were reported at 3 and 6 months (Table 2), particularly in physical functioning, role limitations and pain, while the components of mental health QOL that improved more significantly were vitality and social functioning. Tables 3 and 4 report the results of groups $\mathrm{A}$ and $\mathrm{B}$, respectively, for each domain of the SF-36.

Table 1: Subjects' characteristics.

\begin{tabular}{|l|c|c|}
\hline & GROUP A & GROUP B (PLACEBO) \\
\hline Age, Years, Mean (SD) & $70(65-78)$ & $73(67-83)$ \\
\hline Height $(\mathrm{m})$ & 1.57 & 1.61 \\
\hline Weight $(\mathrm{kg})$ & 70,64 & 73,86 \\
\hline
\end{tabular}

Table 2: The SF-36 score of the 2 groups at baseline, at 3 and 6 months.

\begin{tabular}{|l|c|c|c|}
\hline & GROUP A & GROUP B & P $^{*}$ \\
\hline SF-36 (WEEK 0) & $50.3 \pm 11.24$ & $49.6 \pm 9.3$ & 0.001 \\
\hline SF-36 (WEEK 12) & $53.3 \pm 7.03$ & $46.5 \pm 11.24$ & 0.001 \\
\hline SF-36 (WEEK 24) & $55.4 \pm 8.1$ & $47.69 \pm 10.8$ & 0.001 \\
\hline
\end{tabular}

Table 3: SF-36 domains in group A.

\begin{tabular}{|l|c|c|c|}
\hline SF-36 & 0 month & 3 month & 6 months \\
\hline Physical functioning & $40.0[20.0 ; 60.0]$ & $47.5[43.0 ; 65.0]^{*}$ & $52.5[45.0 ; 61.0]^{*}$ \\
\hline Physical role & $25.0[00.0 ; 50.0]$ & $30.5[02.0 ; 120.0]^{*}$ & $37.5[00.0 ; 100.0]$ \\
\hline Bodily pain & $31.0[22.0 ; 41.0]$ & $38.5[38.0 ; 42.0]^{*}$ & $41.5[41.0 ; 50.0]^{*}$ \\
\hline General health & $48.5[22.0 ; 77.0]$ & $50.0[42.0 ; 72.0$ & $52.0[43.0 ; 62.0]$ \\
\hline Vitality & $27.5[15.0 ; 40.0]$ & $35.0[30.0 ; 40.0]^{*}$ & $45.0[40.0 ; 60.0]^{*}$ \\
\hline Social function & $50.0[25.0 ; 75.0]$ & $53.5[50.0 ; 65.0]$ & $55.5[50.0 ; 75.0]$ \\
\hline Emotional role & $33.3[00.0 ; 66.7]$ & $33.3[00.0 ; 100.0]$ & $33.3[00.0 ; 100.0]$ \\
\hline Mental health & $60.0[28.0 ; 76.0]$ & $66.0[48.0 ; 78.0]^{*}$ & $72.0[55.0 ; 80.0]^{*}$ \\
\hline
\end{tabular}

${ }^{\star}$ Statistically significant $(\mathrm{p}=0.001)$.

Table 4: SF-36 domains in group B.

\begin{tabular}{|l|c|c|c|}
\hline SF-36 & 0 month & 3 months & 6 months \\
\hline Physical functioning & $38.0[20.0 ; 60.0]$ & $40.5[43.0 ; 65.0]$ & $40.5[45.0 ; 61.0]$ \\
\hline Physical role & $27.0[00.0 ; 50.0]$ & $30.5[02.0 ; 120.0]$ & $31.5[00.0 ; 100.0]$ \\
\hline Bodily pain & $29.0[22.0 ; 41.0]$ & $30.5[38.0 ; 42.0]$ & $33.5[41.0 ; 50.0]$ \\
\hline General health & $50.5[22.0 ; 77.0]$ & $52.0[42.0 ; 72.0]$ & $52.0[43.0 ; 62.0]$ \\
\hline Vitality & $30.5[15.0 ; 40.0]$ & $33.0[30.0 ; 40.0]$ & $34.0[40.0 ; 60.0]$ \\
\hline Social function & $47.0[25.0 ; 75.0]$ & $47.5[50.0 ; 65.0]$ & $46.5[50.0 ; 75.0]$ \\
\hline Emotional role & $31.3[00.0 ; 66.7]$ & $33.3[00.0 ; 100.0]$ & $32.3[00.0 ; 100.0]$ \\
\hline Mental health & $58.0[28.0 ; 76.0]$ & $60.0[48.0 ; 78.0]$ & $61.0[55.0 ; 80.0]$ \\
\hline
\end{tabular}


There were no significant differences between group $A$ and group B in mean HADS-A or HADS-D scores at baseline or during follow-up. However, after three months of follow-up there was a trend towards a reduction in the mean HADS-A score in group A compared to group $\mathrm{B}$, resulting in a significant difference in mean change: -0.9 $(-1.8,-0.01)$ in group A versus $0.5(-0.4$ to 1.4$)$ in group $B, p=0.02$. Moreover, after 6 months of follow-up the HADS-D scores remained stable in group A, but tended to increase in group B, resulting in a significant difference in the variation of score mean during this period: $0.05(-0.8,0.9)$ in group A versus $1.0(0.3-1.8)$ in group B (p $=0.03$ ) (Table 5). Significant improvements were also observed in the PSQI score of the both study groups but in group A the difference was statistically significant $(\mathrm{p}=0.002)$ (Figure 1$)$.

\section{Discussion}

Rice bran oryzalose exerts immunomodulating effects, which include upregulation of natural killer (NK) cell activity, increase of phagocytic cell functions, modulation of cytokines production and promotion of $\mathrm{T}$ and B lymphocyte proliferation [22]. The remaining components that are resistant to digestion serve as prebiotics for the gut microbiota, which induces anti-inflammatory and immunomodulatory effects and influence behavioral changes across the gut-brain axis. Among the large number of natural agents derived from plants and employed in the integrative management of cancer patients, oryzalose is extremely promising, due to its effectiveness in improving the clinical status of patients [23-26]. QoL improvements (sleep, appetite, digestion, physical activity, anxiety and pain), as well as reduced adverse effects during cancer therapy, have been reported in several studies [27-35]. Supplementating with oryzalose ( $400 \mathrm{mg} / \mathrm{die}$ ) for three months also significantly enhanced the QoL scores of healthy elderly adults in a randomized controlled trial [36]. Clinical research on the effects of oryzalose in cancer patients is still in its early

Table 5: Mean HADS score in the 2 groups before and after oryzalose.

\begin{tabular}{|l|c|c|}
\hline & GROUP A & GROUP B \\
\hline HADS-A (WEEK 0) & $6.5(5.46$ to 7.4$)$ & $6.0(6.7$ to 7.5$)$ \\
\hline HADS-D (WEEK 0) & $6.1(5.4$ to 6.7$)$ & $6.0(5.5$ to 6.5$)$ \\
\hline HADS-A (WEEK 12) & $6.3(5.8$ to 6.8$)$ & $6.2(6.8$ to 7.6$)$ \\
\hline HADS-D (WEEK 12) & $5.9(5.3$ to 6.5$)$ & $6.0(5.8$ to 6.8$)$ \\
\hline HADS-A (WEEK 24) & $6.0(5.5$ to 6.5$)$ & $6.3(6.7$ to 7.3$)$ \\
\hline HADS-D (WEEK 24) & $5.7(5.3$ to 6.4$)$ & $6.2(5.6$ to 6.6$)$ \\
\hline
\end{tabular}

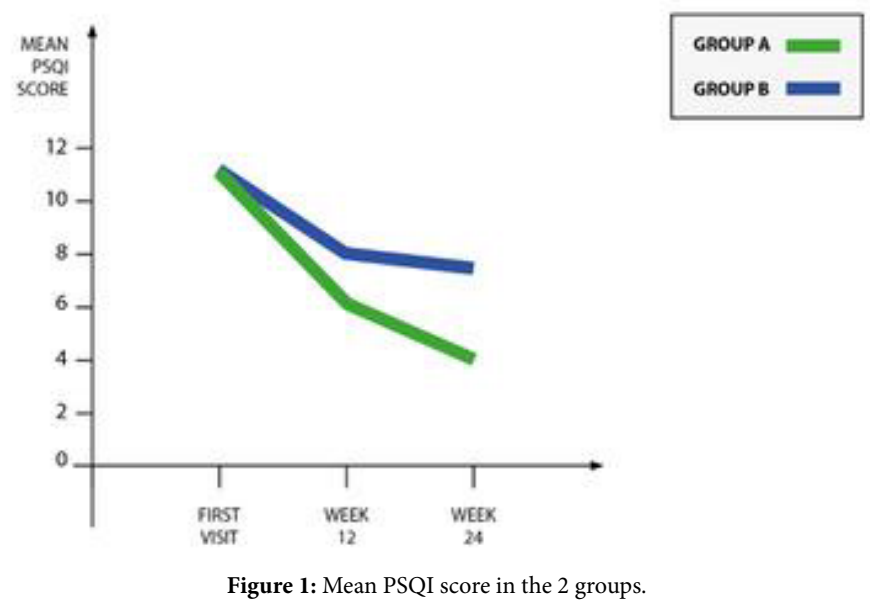

stage [37-39], and most of the trials have several limitations, unclear risks of bias, non-validated QoL measurements [40,41]. Furthermore, none of these trials attempted to rule out the impact of placebo in QoL results. The gut microbiota is achieving increasing attention as a powerful regulator of quality of life, sleep and psychological outcomes in cancer patients; moreover, microbiome composition may be modulated by diet, exercise, behaviours, xenobiotics and probiotics [42-46]. Among the most studied and widely used probiotics, Lactobacillus plantarum is an excellent candidate for supplementation, due to its resistance to many classes of antibiotics and anti-inflammatory properties [47]. In this study, we aimed at assessing the effect of the prebiotic oryzalose in association with the probiotic Lactobacillum Plantarum in the management of cancerrelated side effects and quality of life of breast cancer patients undergoing hormonal therapy. Several limitations of our study require consideration. First, we carried out this study in only two academic cancer center, in a sample of patients with limited racial and ethnic diversity; therefore, our findings cannot be generalized to other more heterogeneous populations. In addition, the short-term follow-up of the enrolled patients could be considered as another limitation of the study. On of the major strenghs of the study is the advanced age of the population enrolled (over 65), who are usually excluded from clinical trials, despite being the most affected by the disease, due to the complexity of clinical issues [48]. Despite this, our drop out rate and participants compliance to the protocol were excellent, showing high profiles of safety for the compound under investigation. The results of this feasibility trial will inform the planning of a larger clinical trial for definitive conclusions.

\section{Conclusion}

This study showed an excellent compliance to the protocol of treatment and, as preliminary results, improved quality of life in terms of physical functioning, pain, vitality and psychological well-being in elderly breast cancer patients in the treatment arm compared to placebo. Further similar studies with longer follow-up periods in breast cancer patients are warranted in order to explore the impact of symbiotics and other modulators of patients' microbiome on cancer-related symptoms and quality of life, even in elderly populations due to the high adherence and safety profile of the prebiotic and probiotic treatment.

\section{Conflict of Interest}

The authors declare they have no competing interests.

\section{References}

1. Britti MS, Roselli M, Finamore A, Merendino N, Mengheri E (2006) Regulation of immune response at intestinal and peripheral sites by probiotics. Biologia 61: 735-740.

2. Francescone R, Hou V, Grivennikov, SI (2014) Microbiome, Infammation, and Cancer. Cancer J 20: 181-189. [crossref]

3. Kwa M, Plottel CS, Blaser MJ, Adams S (2016) The Intestinal Microbiome and Estrogen Receptor-Positive Female Breast Cancer. J Natl Cancer Inst 108. [crossref]

4. Guo M, Liu T, Li P, Wang T, Zeng C, et al. (2019) Association Between Metabolic Syndrome and Breast Cancer Risk: An Updated Meta-Analysis of Follow-Up Studies. Front Oncol 9. [crossref]

5. Baker JM, Al-Nakkash L, Herbst-Kralovetz MM (2017) Estrogen-gut microbiome axis: Physiological and clinical implications. Maturitas 103: 45-53. [crossref]

6. Valles-Colomer M, Falony G, Darzi Y, Tigchelaar EF, Wang J, et al. (2019) The neuroactive potential of the human gut microbiota in quality of life and depression. Nat Microbiol 4: 623-632. [crossref] 
7. Costantini L., Magno S., Albanese D, Claudio Donati, Romina Molinari, et al. (2018) Characterization of human breast tissue microbiota from core needle biopsies through the analysis of multi hypervariable 16S-rRNA gene regions. Scientifica Reports 8.

8. Katherine MH, James AF, Larry JF, Ursel MES, Daniel LB, et al. (2011) Characterization of the diversity and temporal stability of bacterial communities in human milk. PLos One 6. [crossref]

9. Raul CR, Carmen C, Kirsi L, Seppo S, Erika Isolauri, et al. (2012) The human milk microbiome changes over lactation and is shaped by maternal weight and mode of delivery. Am. J. Clin. Nutr 96: 544-551. [crossref]

10. Bhatt AP, Redinbo MR, Bultman SJ (2017) Te role of the microbiome in cancer development and therapy: Microbiome and Cancer. CA Cancer J. Clin 67: 326-344. [crossref]

11. Gopalakrishnan V, Spencer CN, Nezi L, Reuben A, Andrews MC, et al. (2018) Gut microbiome modulates response to anti-PD-1 immunotherapy in melanoma patients. Science 359: 97-103. [crossref]

12. Routy B, Le Chatelier E, Derosa L, Duong CPM, Alou MT, et al. (2018) Gut microbiome influences efficacy of PD-1-based immunotherapy against epithelial tumors. Science 359: 91-97. [crossref]

13. Roy S, Trinchieri G (2017) Microbiota: a key orchestrator of cancer therapy. Nat Rev Cancer 17: 271-285. [crossref]

14. McQuade JL, Daniel CR, Helmink BA, Wargo JA (2019) Modulating the microbiome to improve therapeutic response in cancer. Lancet Oncol 20.

15. Lissoni P, Messina G, Lissoni A, Franco R (2017) The psychoneuroendocrineimmunotherapy of cancer: Historical evolution and clinical results. J Res Med Sci 26. [crossref]

16. Lissoni P, Rovelli F (2012) Principles of psychoneuroendocrinoimmunotherapy of cancer. Immunotherapy 4:77-86. [crossref]

17. Messina G, Lissoni P, Bartolacelli E, Magotti L, Clerici M, et al. (2010) Relationship between psychoncology and psychoneuroendocrinoimmunology (PNEI): enhanced T-regulatory lymphocyte activity in cancer patients with self-punishement, evaluated by Rorschach test. In Vivo, 24: 75-78. [crossref]

18. McSorley ST, Dolan RD, Roxburgh CSD, McMillan DC, et al. (2017) How and why systemic inflammation worsens quality of life in patients with advanced cancer. Expert Rev Qual. Life Cancer Care 2: 167-175.

19. Laird BJA, McMillan DC, Fayers P, Fearon K, Kaasa S, et al. (2013) The systemic inflammatory response and its relationship to pain and other symptoms in advanced cancer. Oncol 18: 1050-1055. [crossref]

20. Liang OS, Pak SC, Micalos PS, Emily Schupfer, Rob Zielinski, et al. (2020) Rice brain arabinoxylan compound and quality of life of cancer patients: study protocol for a randomized pilot feasibility trial. Contemporary Clinical Trials Communications 19. [crossref]

21. Hays RD, Sherbourne CD, Mazel RM. (1993) The RAND 36-Item Health Survey 1.0. Health Econ 2: 217-227. [crossref]

22. Mantovani A, Allavena P, Sica A, Balkwill F (2008) Cancer-related inflammation. Nature 454: 436-444.

23. Hajtò T, Horvath l, Baranyai L, Kuzma M, Perjesi P (2016) Can the EGFR inhibitors increase the immunomodulatory effects of standardized plant extracts (mistletoe lectin and arabinoxylan) with clinical benefit? Case report of a patient with lung adenocarcinoma. Clin Case Rep Rev 2: 456-459.

24. Meshitsuka KA (2013) case of stage IV hepatocellular carcinoma treated by KM900. Biobran and psychotherapy has presented significant good results. Pers Med Universe (Japanese Ed.) 1: 46-48.

25. Elsaid AF, Fahmi RM, Shaheen M, Ghoneum M (2020) The enhancing effects of Biobran/ MGN - 3, an arabinoxylan rice bran, on healthy old adults' health-related quality of life: a randomized, double-blind, placebo-controlled clinical trial. Qual. Life Res 29: 357-367. [crossref]

26. Masood AI, Sheikh R, Anwer RA (2013) "BIOBRAN MGN-3"; Effect of reducing side effects of chemotherapy in breast cancer patients. Prof Med J 20: 13-16.

27. Colotta F, Allavena P, Sica A, Garlanda C, Mantovani A (2009) Cancer-related inflammation, the seventh hallmark of cancer: links to genetic instability. Carcinogenesis 30: 1073-1081. [crossref]

28. McAllister SS, Weinberg RA (2014) The tumour-induced systemic environment as a critical regulator of cancer progression and metastasis. Nat. Cell Biol 16: 717 -727. [crossref]

29. U. Jaffer, R. G. Wade, T. (2010) Gourlay. Cytokines in the systemic inflammatory response syndrome: a review. HSR Proc. Intensive Care Cardiovasc. Anesth 2: 161 -175.

30. Santos JC, Pyter LM (2018) Neuroimmunology of behavioral comorbidities associated with cancer and cancer treatments. Front. Immunol 9. [crossref]

31. Ghoneum M, Agrawal S (2011) Activation of human monocyte-derived dendritic cells in vitro by the biological response modifier arabinoxylan rice bran (MGN-3/Biobran). Int J Immunopathol Pharmacol 24: 941-948. [crossref]

32. Ghoneum M, Gollapudi S (2003) Modified arabinoxylan rice bran (MGN - 3/ Biobran) sensitizes human $\mathrm{T}$ cell leukemia cells to death receptor (CD95)-induced apoptosis. Canc. Lett 201: 41-49. [crossref]

33. Pérez Martínez A, Valentín J, Fernández J, Hernández-Jiménez E, López-Collazo $\mathrm{E}$ et al. (2015) Arabinoxylan rice bran (MGN - 3/ Biobran) enhances natural killer cellmediated cytotoxicity against neuroblastoma in vitro and in vivo. Cytotherapy 17: 601 -612. [crossref]

34. Ghoneum M (2016) From bench to bedside: the growing use of arabinoxylan rice bran (MGN - 3/ Biobran) in cancer immunotherapy. Austin Immunol 1.

35. Mendis M, Leclerc E (2016) Simsek. Arabinoxylans, gut microbiota and immunity. Carbohydr Polym 139: 159 -166. [crossref]

36. Ooi SL, McMullen SL, Golombick T, Pak SC (2018) Evidence-based review of BioBran/ MGN-3 arabinoxylan compound as a complementary therapy for conventional cancer treatment. Integr. Canc. Ther 17: 165 -178. [crossref]

37. Kawai T (2004) One case of a patient with umbilical metastasis of recurrent cancer (Sister Mary Joseph's Nodule, SMJN) who has survived for a long time under immunomodulatory supplement therapy. Clin Pharmacol Ther 14: 281-288.

38. Kaketani (2014) A case where an immunomodulatory food was effective in conservative therapy for progressive terminal pancreatic cancer. Clin. Pharmacol. Ther 14: 273-279.

39. Okamura Y (2004) The clinical significance of modified arabinoxylan from rice bran (BioBran/ MGN - 3) in immunotherapy for cancer. Clin Pharmacol Ther 14: 289-294. [crossref]

40. Markus J, Miller A, Smith M (2006) Orengo. Metastatic hemangiopericytoma of the skin treated with wide local excision and MGN - 3. Dermatol Surg 32: 145-147. [crossref]

41. Hajto T, Baranyai L, Kirsch A, Kuzma M, Perjési P (2015) Can a synergistic activation of pattern recognition receptors by plant immunomodulators enhance the effect of oncologic therapy? Case report of a patient with uterus and ovary sarcoma, Clin. Case Rep. Rev 1: $235-238$.

42. Adlercreutz H (1990) Western diet and Western diseases: Some hormonal and biochemical mechanisms and associations. Scand. J. Clin. Lab. Investig. Suppl 201: 3-23. [crossref]

43. Keku TO, Dulal S, Deveaux A, Jovov B, Han X (2015) The gastrointestinal microbiota and colorectal cancer. Am J Physiol Gastrointest Liver Physiol 308. [crossref]

44. Turnbaugh PJ, Hamady M, Yatsunenko T, Cantarel BL, Duncan A, et al. (2009) A core gut microbiome in obese and lean twins. Nature 457: 480-484. [crossref]

45. Mikó E, Kovács T, Sebő É, Tóth J, Csonka T, et al. (2019) Microbiome-Microbial Metabolome-Cancer Cell Interactions in Breast Cancer-Familiar, but Unexplored. Cells 8. [crossref]

46. Parida S, Sharma D (2019) The Microbiome-Estrogen Connection and Breast Cancer Risk. Cells 8. [crossref]

47. Petrovics, Szigeti G, Hamvas S, Máté A, Betlehem J, Hegyi G (2016) Controlled pilot study for cancer patients suffering from chronic fatigue syndrome due to chemotherapy treated with BioBran (MGN-3- Arabinoxylane) and targeted radiofrequency heat therapy. Eur J Integr Med 8: 29-35.

48. Sedrak M, Freedman R, Cohen H, Muss H, et al. (2020) Older adult participation in cancer clinical trials: a systematic review of barriers and interventions. Ca Cancer J Clin 71.

\section{Citation:}

Rossi C, Filippone A, Rossi MM, Guarino D, Magno S, et al. (2021) Effects of a Symbiotic on the Quality of Life of Elderly Patients with Breast Cancer: a Randomized Controlled Pilot Trial. Cancer Stud Ther J Volume 6(1): 1-4 